\title{
A case study from the Memorial Sloan Kettering Cancer Center: A 40-year-old woman with PET detected mediastinal disease at presentation
}

\author{
B. McCormick, G. D’Andrea, M. Spierer \\ Memorial Sloan Kettering Cancer Center, New York, NY, USA
}

\section{Diagnosis and presentation}

This is a 40-year-old woman, who noted a mass in the left axilla and a 'shooting' pain in her left breast. Her local gynaecologist sent her for a screening bilateral mammogram, revealing only of an enlarged left axillary node. An ultrasound done at the same time documented a suspicious mass in the upper inner quadrant of the left breast, measuring $3.3 \mathrm{~cm}$ in greatest diameter. Left axillary adenopathy was also noted on ultrasound.

Next, the patient saw a local surgeon, who performed a lumpectomy of the breast mass. The pathology upon review at Memorial Hospital revealed a $1.1 \mathrm{~cm}$ invasive ductal carcinoma, with both histologic and nuclear grades of III/III. High-grade ductal carcinoma in situ was also present, but comprised less than $25 \%$ of the total tumour mass. Vascular invasion and extensive lymphatic permeation were observed. Although the margins were negative, multiple lymphatic tumour emboli were noted close to the anterior margin. Estrogen receptor (ER), progesterone receptor (PR) and human epidermal growth factor receptor 2(Her-2) neu markers were all negative.

The patient came to Memorial Hospital for further evaluation. Clinically, her examination was notable only for the healing biopsy site in the left breast and at least three distinct lymph nodes palpable in the left axilla, the largest of which measured $3 \mathrm{~cm}$. Her extent of disease work-up included a bone scan, which was negative, and a computed tomography

Correspondence to: Beryl McCormick, MD, Department of Radiation Oncology, Memorial Sloan Kettering Cancer Center, 1275 York Avenue, New York, NY 10021 USA. E-mail: mccormib@mskcc.org; Tel: +1212639 6828; Fax: +1212 6398876

Publication date 28/01/05 BCO/337/2004/CS

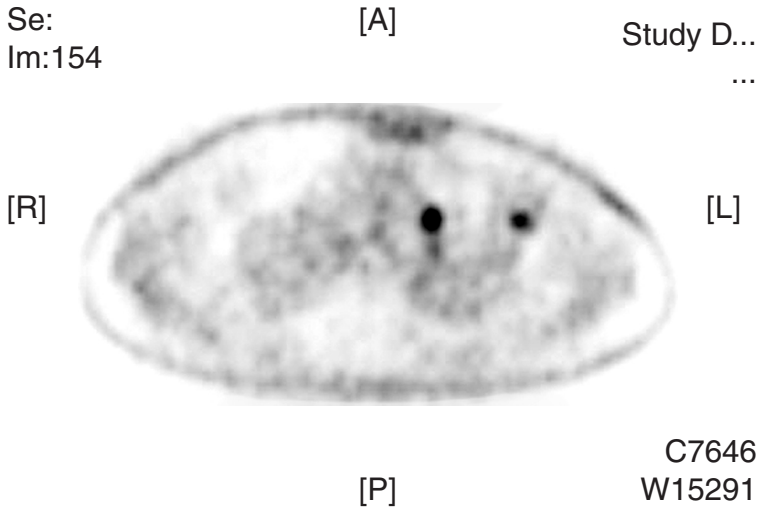

Figure 1.

Pre-chemotherapy PET scan showing uptake in both the left axilla and pre-vascular region.

(CT) scan of the chest, abdomen and pelvis, which demonstrated left axillary and internal mammary adenopathy, and an enlarged anterior mediastinal node measuring 3.3 by $1.9 \mathrm{~cm}$. A positron emission tomography (PET)/CT scan confirmed abnormal [F-18]2-deoxy-2-fluoro-D-glucose (FDG) uptake in the left axilla (standardized uptake value (SUV) 4.2), left internal mammary node region, and the prevascular region. A bilateral breast magnetic resonance imaging (MRI) revealed only post-operative changes in the left breast (Fig. 1).

The patient had no family history of breast cancer, and no significant medical or surgical history, except for cosmetic surgery including a bilateral breast reduction mammoplasty in 1997.

\section{Treatment and outcome}

The patient was taken to the operating room by a thoracic surgeon, for a Chamberlain procedure; the 
suspicious anterior mediastinal nodes proved to be consistent with metastatic breast carcinoma. She was staged as a Stage IV case, and at that time, the patient was offered treatment with weekly Taxol, at a dose of $80 \mathrm{mg} / \mathrm{m}^{2}$. After 10 cycles, the patient was re-scanned and noted to have a modest partial response. Taxol was continued for another 10 cycles, with stability noted on the next round of imaging studies. As both her modest response to Taxol and her concern with her hair loss, her chemotherapy was then switched to Xeloda, $1500 \mathrm{mg}$ BID. Three days after her first cycle, she developed a skin rash after exposure to the sun on her face, upper chest and arms, and the chemotherapy was stopped. She was referred to dermatology, and treated with topical Diprolene. She had resolution of the rash.

She was next referred for consideration of local surgery and/or radiation of her persistent local disease. On examination, the patient had a Karnofsky performance status (KPS) of $90 \%$, and was asymptomatic. Her clinical examination revealed complete resolution of the palpable disease in her left axilla, and good healing of her breast biopsy site. Another set of imaging studies continued to reveal persistent adenopathy in the left axilla, left internal mammary node region, and the anterior mediastinum.

She was discussed in detail at the Breast Service multi-disciplinary conference.

\section{Comments}

The patient was asymptomatic; thus, advising any local regional treatment with either surgery or radiation was discussed within that context. The thoracic surgery service had previously decided not to attempt to resect her mediastinal disease. There was complete consensus amongst the clinicians from both surgery and radiation oncology that neither breast and axillary surgery nor radiation treatments directed to those areas would be appropriate at this time, given her stage and her lack of any symptoms. The extent of her disease at diagnosis would dictate a large and complex radiation field, to address all areas of disease. Given the remaining size of her disease on recent imaging, the expectation of success of such radiation in terms of long-term control was quite low. A surgical procedure was similarly deemed not appropriate at this point in her disease management. At such time when she exhibits progression, palliative local treatment will be reconsidered to address her complaints and symptoms at that time.

Further systemic treatment was advised by the medical oncology group, given her negative marker status. The patient was scheduled to re-start her Xeloda treatments. 